\title{
Prevention of paediatric respiratory tract infections: emphasis on the role of OM-85
}

\author{
U.B. Schaad
}

\begin{abstract}
The burden of respiratory tract infections in paediatrics is extremely high, in both industrialised and developing countries. Because adequate diagnosis and causative therapies of these often recurrent respiratory tract infections bear substantial limits, preventive measures deserve priority. The mainstays are parent education, active immunisation strategies and nonspecific immunostimulation with bacterial products.

This article summarises the five key studies on the use of the immunoactive agent OM-85 (Broncho-Vaxom) for prevention of recurrent respiratory tract infections in children. Such bacterial immunostimulants are especially indicated for young infants and children who are known and/or expected to suffer from at least three respiratory tract infections per winter season.
\end{abstract}

KEYWORDS: Bacterial immunostimulants, immunostimulation, paediatrics, prevention, respiratory tract infections

D) aediatric acute infections of the airways continue to play an important role worldwide. The burden of these diseases is reflected by: 1) high incidence; 2) substantial morbidity and potential sequelae; 3) tendency of overdiagnosis, especially in streptococcal pharyngitis and acute otitis media; 4) associated overuse and misuse of antibiotics; and 5) relevant contributor to healthcare costs and indirect societal costs.

Nearly $50 \%$ of the paediatric consultations in Switzerland and other industrialised countries are caused by respiratory tract infections (RTIs) $[1,2]$. Acute RTIs are among the leading causes of childhood mortality [3]. The proportion of deaths directly attributable to acute RTIs varies between $23 \%$ in countries with high child mortality and $15 \%$ in countries with low child mortality. For the year 2000 it has been calculated that throughout the world 1.9 million (95\% CI, 1.6-2.2 million) children died from acute RTIs, $70 \%$ in Africa and south-east Asia.

The vast majority (80-90\%) of these infections are caused by viruses. Uncomplicated upper respiratory tract infections (URTIs) are usually selflimiting and do not require antibiotics. However, several bacterial complications can arise, such as acute otitis media, sinusitis and bronchitis. In a French study, overall incidence of complications was $16.8 \%(10.9 \%$ acute otitis media, $4 \%$ laryngitis or bronchitis, $1.9 \%$ pulmonary infections) [4].

The development and spread of antibacterial resistance in bacteria that commonly cause community-acquired RTIs is a major global healthcare problem [5]. Unnecessary antibiotic therapy (overuse) and poor therapeutic choice, dosage and/or duration (misuse) both contribute to bacterial resistance, avoidable toxicity and increased costs. Hence, improving antibacterial use in childhood RTIs is an important challenge for the physicians in charge [6].

\section{PATHOGENESIS}

The high incidence of RTIs in young infants and preschool children is explained by: 1) increased exposure to respiratory pathogens by siblings and in child care; 2) environmental factors; 3 ) defects in the immune system.

Crowded conditions, such as those found in daycare settings, favour the colonisation and spread of pathogens causing RTIs [7]. Well documented environmental risk factors include passive smoking, exposure to pollutants and absence of breastfeeding [8]. Familial predisposition is a risk factor for recurrent and severe disease. This genetic predisposition seems associated with anatomical, physiological and/or immunological features [7].

Defects in the immune systems are well known to be linked with frequent RTIs. It has been shown that $57 \%$ of children with recurrent RTIs (at least three episodes a year during at least 2 yrs) were deficient in one of the immunoglobulin (Ig)G subclasses and that $17 \%$ were IgA deficient [9]. IgG subclass deficiency is quite prominent in young children but rare in older children, suggesting a transient immaturity of the immune
CORRESPONDENCE

U.B. Schaad

University Children's Hospital

P.O. Box

$\mathrm{CH}-4002$ Basel

Switzerland

Fax: 41616856001

E-mail: urs-b.schaad@unibas.ch 
system as one of the possible pathogenic factors. Defects in the immune system, such as common variable immunodeficiency and the more frequent selective IgA deficiency, are known to be linked with frequent RTIs caused by bacteria and viruses [10].

\section{PREVENTION}

The strategies for prevention of RTIs in children are: 1) parent education (normal prevalence, familial predisposition, risk factor modification, natural course without and with antibiotics); 2) specific immunisation; 3) chemoprophylaxis; 4) surgery; 5) oligosaccharides; and 6) specific and nonspecific immunostimulation.

Vaccines ultimately represent the best opportunity to reduce the morbidity and mortality associated with paediatric RTIs. It is beyond the scope of this article to review the promising data on pneumococcal conjugate, influenza and other vaccines.

Currently, antibacterial chemoprophylaxis is only exceptionally appropriate. The benefit is modest and the risk of selective pressure of the antibacterial drug for emergence of resistant pathogens is real $[7,11]$. The data on antiviral chemoprophylaxis during influenza in children are preliminary and conflicting.

Many trials and reviews attempted to define the role of ear, nose and throat surgery (e.g. tonsillectomy, adenoidectomy, myringotomy with/without placement of tympanostomy tubes) in the prevention of RTIs in paediatric patients [12, 13]. This is another topic where there is more art than science!

Oligosaccharides were demonstrated in vitro and in animal experiments to prevent bacterial mucosal attachment and colonisation, and infection [14]. The role of xylitol in prevention of childhood acute otitis media remains controversial [7].

The correlation between recurrent RTIs and immunological deficiencies mentioned above $[9,10]$ represents the rationale for a nonspecific immunostimulating preventive treatment of patients suffering from recurrent RTIs.

\section{BACTERIAL IMMUNOSTIMULANTS}

There is a large body of immunological data and clinical experience in children with four such products, developed and introduced 3-4 decades ago.

Biostim ${ }^{2}$ (Sanofi Aventis, Paris, France) is a glycoprotein extract from Klebsiella pneumoniae.

Ribomunyl ${ }$ (Pierre Fabre, Castres, France) contains ribosomes from four bacterial species (Haemophilus influenzae, Streptococcus pyogenes, Streptococcus pneumoniae, K. pneumoniae) and K. pneumoniae membrane proteoglycans. Bacterial ribosomes have been shown to be highly immunogenic, whereas bacterial membrane proteoglycans act as adjuvants.

I.R.S. $19{ }_{\circledast}$ (Solvay, Brussels, Belgium) is a nasal spray suspension of lysates of 19 respiratory bacteria.

Broncho-Vaxom ${ }^{\circledR}$ (OM-85) (OM PHARMA, Meyrin-Geneva, Switzerland) is a lyophilised extract from eight respiratory bacteria (H. influenzae, Diplococcus pneumoniae, K. pneumoniae, Klebsiella ozaenae, Staphylococcus aureus, S. pyogenes, Streptococcus viridans and Moraxella catarrhalis).
Many in vitro and in vivo studies indicate that these bacterial products stimulate the local immune responses and possibly systemic immune responses. These actions can be summarised as pleiotropic immunomodulating effects, i.e. activating different systems in the chain of immunological defence reactions [15].

The majority of clinical trials with bacterial immunostimulants showed excellent safety and reduction of RTIs, sometimes accompanied by a reduction in antibiotics and other concomitant medications. Among these reports, there are randomised, double-blind, placebo-controlled trials that comply, at least in its essentials, with modern good clinical practice standards; such quality regarding study design, conductance and analysis entitles the connotation "key study". Review of the literature revealed five such key studies for OM-85 (Broncho-Vaxom) use in paediatric patients.

The next section of this article summarises the five key studies on the use of OM-85 (Broncho-Vaxom) for prevention of RTIs in children with acute recurrent RTIs [16-20].

\section{CLINICAL KEY STUDIES WITH OM-85 (BRONCHO-VAXOM)}

These reports published in peer-reviewed medical journals deal with randomised, double-blind, placebo-controlled trials in paediatric patients with frequent RTIs, especially URTIs, i.e. prone to continue to develop recurrent RTIs; details on publications, authors and study patients are shown in table 1.

The main study end-point was prevention of recurrent RTIs, measured by number, type, severity and duration of RTIs, and additionally documented by consumption of antibiotics and other drugs, and by absenteeism from school. Also, reportings on adverse events were adequate allowing reliable safety evaluation.

SCHAAD et al. [16] conducted a randomised, double-blind, placebo-controlled, multicentre study with OM-85 in 220 patients aged 36-96 months, suffering from recurrent URTIs. Treatment was one capsule daily during month 1 and during 10 days in months $3-5$. URTI was defined by the presence of at least two of the following: rhinitis, pharyngitis, cough, hoarseness, fever $\geqslant 38.5^{\circ} \mathrm{C}$, or URTI-related prescription of an antibiotic. OM-85-treated patients had a lower rate of URTIs $(\mathrm{p}<0.05)$, as compared with patients receiving placebo. The cumulated difference in URTIs between the two groups reached -0.40 URTIs per patient in 6 months, corresponding to a statistically significant $16 \%$ reduction in the active group with respect to placebo. The largest difference was observed in the patients having had at least three URTIs during the study period. The difference between OM-85 and placebo was independent of age but was more important in patients reporting a larger number of URTIs in the previous year. Safety and tolerance of test medication were good, comparable with placebo.

Substantially greater reductions of URTIs, by $52 \%$ and $38 \%$, respectively, have been reported with OM-85 in comparison to placebo during two double-blind studies from Mexico [17, 18]. These more marked findings with respect to those found in the Swiss/German trial [16] may be linked to differences in settings. Indeed, one trial included only females aged 6-13 yrs, living in a fairly crowded centre favouring microbial 


\begin{tabular}{|c|c|c|c|c|c|}
\hline \multirow[t]{2}{*}{ Reference } & \multirow[t]{2}{*}{ Year } & \multirow[t]{2}{*}{ Country } & \multicolumn{3}{|c|}{ Study population } \\
\hline & & & Age range yrs & No OM-85 & No placebo \\
\hline Schaad [16] & 2002 & Switzerland/Germany & $3-8$ & 120 & 100 \\
\hline Gutiérrez-Tarango [18] & 2001 & Mexico & $1-12$ & 26 & 28 \\
\hline Collet [19] & 1993 & France & $0.5-2$ & 210 & 213 \\
\hline Paupe [20] & 1991 & France & $0.5-19$ & 61 & 55 \\
\hline
\end{tabular}

contamination (an orphanage), located in a region with high air pollution (Mexico City), and who were all highly susceptible to RTIs (median of five infections per child over the previous 6 months) [17]. Overall, the same comments may address the other trial, in particular air pollution and highly susceptible children aged 1-12 yrs with a past mean rate of 12 RTIs during the 12 months prior to entering the study [18].

COLLET et al. [19] addressed the question of the efficacy of OM85 in the primary prevention of recurrent URTIs in 423 young children (age range, 6-12 months) in day-care centres. He found a $48 \%$ reduction of the relative risk of having at least three URTIs after three months of treatment.

The efficacy of OM-85 in breaking the cycle of recurrent infections was also demonstrated by PAUPE [20] in 116 children, aged 6 months to 19 yrs, who had a history of at least three episodes of RTIs in the previous 6-month period. At the end of the prospective study period ( 3 months of treatment plus 3 months of follow-up), the incidence of RTIs in the active group was $35 \%(p<0.01)$ lower than in the placebo group. This effect was more pronounced in children aged $<6$ yrs.

The results of these five key paediatric studies [16-20] (table 1) show that OM-85 diminishes the risk of a RTI (especially URTI) in children with a history of frequent RTIs. This beneficial effect is proportional to the number of RTIs in the past history of the patients and is usually larger in younger children. Risk reduction for young children reporting at least three URTIs, mainly occurring during the cold winter months, may be expected between $20-40 \%$ during preventive treatment with OM-85.

Pharmacoeconomic aspects of prevention of recurrent acute rhinopharyngitis with OM-85 was carefully evaluated by a French expert group using a decision-analysis model [21]. It was concluded that for children reporting at least three URTIs during the previous winter season, a 3-month preventive treatment with OM-85 provides substantial economic benefits both for the insurers and for society in general.

\section{CONCLUSIONS}

The medical and societal burden of acute RTIs in children is enormous.

A special problem are recurrent RTIs due to increased exposure to respiratory pathogens, environmental pollution, and defects in the immune system.
Because causative therapies of recurrent respiratory tract infections bear substantial limits, preventive measures deserve priority. The mainstays are parent education (e.g. risk factors, natural course, role of antimicrobials), active immunisation strategies (e.g. pneumococcal conjugate vaccines, influenza vaccines), and nonspecific immunostimulation with bacterial products. For these immunoactive agents there is proof of efficacy and safety with clearly favourable risk/benefit ratio. Such bacterial immunostimulants are especially indicated for young infants and children who are known and/or expected to suffer from at least three respiratory tract infections per winter season.

\section{REFERENCES}

1 Eggenberger K. Les infections des voies respiratoires les plus fréquentes chez l'enfant dans la pratique ambulatoire [Respiratory tract infections most frequently seen in pediatric outpatient care]. Ars Medici 1993; 83: Suppl. 1, 24-40.

2 Kvaerner KJ, Nafstad P, Jaakkola JJ. Upper respiratory morbidity in preschool children: a cross-sectional study. Arch Otolaryngol Head Neck Surg 2000; 126: 1201-1206.

3 Williams BG, Gouws E, Boschi-Pinto C, Bryce J, Dye C. Estimates of world-wide distribution of child deaths from acute respiratory infections. Lancet Infect Dis 2002; 2: 25-32.

4 Cohen R. Enquête nationale sur les critères de prescription d'une antibiothérapie dans les rhinopharyngites en pédiatrie en ville [National survey regarding antibiotic treatment for pediatric rhino-pharyngitis by private physians (in France)]. Ann Pédiatr (Paris) 1992; 39: 195-201.

5 Felingham D, Feldman C, Hryniewicz W, et al. Surveillance of resistance in bacteria causing community-acquired respiratory tract infections. Clin Microbiol Infect 2002; 8: Suppl. 1, 12-42.

6 Jacobs RF. Judicious use of antibiotics for common pediatric respiratory infections. Pediatr Infect Dis J 2000; 19: 938-943.

7 Klein JO. Nonimmune strategies for prevention of otitis media. Pediatr Infect Dis J 2000; 19: S89-S92.

8 Uhari M, Mäntysaari K, Niemelä M. A meta-analysis of the risk factors for acute otitis media. Clin Infect Dis 1996; 22: 1079-1083.

9 De Baets F, Kint J, Pauwels R, et al. IgG subclass deficiency in children with recurrent bronchitis. Eur J Pediatr 1992; 151: 274-278. 
10 David JR, Rosen FS. Deficiencies in immunoglobulins and cell-mediated immunity (CD-Rom). Chapter IX. Scientific American Medicine (New York NY), SAM-CD, 2000.

11 Williams RL, Chalmers TC, Stange KC, et al. Use of antibiotics in preventing recurrent acute otitis media and in treating otitis media with effusion. A meta-analytic attempt to resolve the brouhaha. JAMA 1993; 370: 1344-1351.

12 Paradise JL, Bluestone CD, Rogers KD, et al. Efficacy of adenoidectomy for recurrent otitis media in children previously treated with tympanostomy tube placement: results of parallel randomized and nonrandomized trials. JAMA 1990; 263: 2066-2073.

13 Paradise JL, Bluestone CD, Colborn DK. Adenoidectomy and adeno-tonsillectomy for recurrent acute otitis media: parallel randomized clinical trials in children not previously treated with tympanostomy tubes. JAMA 1999; 282: 945-953.

14 Zopf D, Roth S. Oligosaccharide anti-infective agents. Lancet 1996; 347: 1017-1021.

15 Emmerich B, Emslander HP, Milatovic D, et al. Effects of a bacterial extract on local immunity of the lung in patients with chronic bronchitis. Lung 1990; 168: 726-731.
16 Schaad UB, Mütterlein R, Goffin $\mathrm{H}$, on behalf of the BVchild study group. Immunostimulation with OM-85 in children with acute recurrent infections of the upper respiratory tract. Chest 2002; 122: 2042-2049.

17 Jara-Pérez J, Berber A. Primary prevention of acute respiratory tract infections in children using a bacterial immunostimulant: a double-masked, placebo-controlled clinical trial. Clin Ther 2000; 22: 748-759.

18 Gutiérrez-Tarango MD, Berber A. Safety and efficacy of two courses of OM-85 BV in the prevention of respiratory tract infections in children during 12 months. Chest 2001; 119: 1742-1748.

19 Collet JP, Ducruet T, Kramer MS, et al. Stimulation of nonspecific immunity to reduce the risk of recurrent infections in children attending day-care centers. Pediatr Infect Dis J 1993; 12: 648-652.

20 Paupe J. Immunotherapy with an oral bacterial extract (OM-85 BV) for upper respiratory infections. Respiration 1991; 58: 150-154.

21 Pessey J-J, Mégas F, Arnould B, Baron-Papillon F. Prevention of recurrent rhinopharyngitis in at-risk children in France; a cost-effectiveness model for a nonspecific immunostimulating bacteral extract (OM-85 BV) Pharmaco-economics 2003; 21: 1053-1068. 